\title{
Developing a new Get Healthy Service program on reducing risky alcohol consumption
}

\section{Ralph Moorea, Nageen Ahmed ${ }^{b}$, Lisa Russellc and Chris Rissellb,d}

a Centre for Population Health, NSW Ministry of Health, Sydney, Australia

${ }^{b}$ NSW Office of Preventive Health, NSW Ministry of Health, Sydney, Australia

${ }^{c}$ Drug and Alcohol Population and Community Programs, NSW Health, Sydney, Australia

${ }^{d}$ Corresponding author: chris.rissel@sswahs.nsw.gov.au

\section{Article history}

Publication date: September 2016 Citation: Moore R, Ahmed N, Russell L, Rissel C. Developing a new Get Healthy Service program on reducing risky alcohol consumption. Public Health Res Pract. 2016;26(4):e2641647. doi: http://dx.doi. org/10.17061/phrp2641647

\section{Key points}

- Telephone coaching can assist adults to improve their lifestyle risk factors

- Assessment of alcohol consumption using the AUDIT-C alcohol questionnaire allows identification of at-risk drinkers who can be referred to relevant clinical pathways

- The Get Healthy Service Alcohol Program is a welcome addition to the range of brief intervention alcohol programs in Australia

\section{Abstract}

Risky alcohol use has significant individual health and social impacts, and is related to short- and long-term harm, including injuries, accidents, liver diseases, some cancers, cardiovascular diseases and alcohol dependence. The Get Healthy Information \& Coaching Service (GHS) is a free telephone coaching service supporting adults 16 years or older to reduce weight, improve nutrition and increase physical activity. Tailored programs are available for Aboriginal people, pregnant women and people at risk of type 2 diabetes. The GHS provides an opportunity to implement a specific program for participants wishing to reduce or cease their alcohol consumption.

This paper describes the processes used to develop an Alcohol Program for the GHS. It outlines the contributions from clinical and program experts, the evidence base for the program's development, clinical screening tools, training for health coaches and referral processes for participants. The Alcohol Program has the potential to provide effective coaching to adults to voluntarily reduce short- and long-term risky alcohol consumption.

\section{Background}

The Australian guidelines to reduce health risks from drinking alcohol (2009) recommend that, for healthy women and men, drinking no more than two standard drinks of alcohol on any given day reduces the lifetime risk of harm from alcohol-related injury or disease (Guideline 1). To reduce the likelihood of risk from a single occasion of drinking, the guidelines recommend consuming four or fewer standard drinks (Guideline 2). ${ }^{1}$ Alcohol consumption has been associated with a range of health-related harms, as well as a high economic cost arising from alcohol misuse. ${ }^{2}$ Collins and Lapsley calculated that the total cost to Australia from alcohol-related harm was $\$ 15.3$ billion in 2004-05. ${ }^{3}$ The New South Wales (NSW) Auditor-General estimated the cost of alcohol-related abuse to NSW Government services at $\$ 1.03$ billion in $2010 .{ }^{4}$

The NSW state health plan: towards 2021 has a stated target of reducing drinking at levels posing lifetime risk (Guideline 1) to below $25 \%$ by $2015 .{ }^{5}$ 
Alcohol abstention is increasing in Australia, with $22 \%$ of the population reporting abstaining from alcohol in 2013, up from $19.9 \%$ in $2010 .{ }^{6}$ Alcohol consumption posing lifetime risk has remained steady, while consumption posing short-term risk has declined from $29.8 \%$ of the population in 2010 to $18.2 \%$ in $2013 .{ }^{6}$ In NSW, risky levels of alcohol consumption have reduced from $29.8 \%$ of the population in 2010 to $27.4 \%$ in 2014 (males $37.1 \%$, females $18.1 \%) .{ }^{7}$ Although the majority of Australians drink at levels that do not pose short-term or lifetime risk, a proportion of people would benefit from reducing their alcohol consumption. ${ }^{7}$

\section{Get Healthy Information \& Coaching Service}

The NSW Get Healthy Information \& Coaching Service (GHS) is a free telephone service, supporting people aged 16 years and older to make changes in relation to physical activity, healthy eating and weight management. The GHS offers up to 10 free coaching calls, where dietitians and/or exercise physiologists use motivational interviewing techniques to set lifestyle modification goals, and identify barriers and solutions for behaviour change. Since 2009, approximately 33000 people have accessed the service. Health outcomes include increased fruit and vegetable intake, reduction in takeaway meals, average weight loss of $3.8 \mathrm{~kg}$ and a waist circumference reduction of $5 \mathrm{~cm}^{8}$ The GHS's tailored programs include an Aboriginal program, a type 2 diabetes prevention program and a healthy gestational weight gain program for pregnant women. Successful implementation of the tailored programs demonstrates the potential for the GHS to focus on reducing unhealthy alcohol consumption.

\section{Feasibility of a GHS Alcohol Program}

Although reducing alcohol consumption has not been a specific priority for the GHS, participants are asked about the frequency and quantity of their alcohol consumption. A review of unpublished GHS data from January 2013 to January 2015 found that $57 \%$ of participants reported not drinking in a usual week when starting the service. ${ }^{9}$ This proportion had increased to $67 \%$ by completion of the GHS program. Additionally, 20\% of participants reported consuming three or more alcoholic beverages on a day when they drank alcohol (Figure 1). This figure reduced to $13 \%$ by completion, suggesting that participants modify alcohol consumption during the course of the program, primarily to reduce kilojoule consumption and lose weight. The percentage of participants who reported consuming four or more drinks on a day they drank alcohol reduced from $8 \%$ at baseline to $4 \%$ by completion of the program (Figure 2). Since 2010, 3930 (13\%) participants reported, at baseline, drinking at levels that placed them at risk of alcohol-related harm. Using these data, the GHS estimates that, each year, 786 participants will be eligible
Figure 1. Get Healthy participants' lifetime risk of harm, based on consumption of $>2$ standard drinks on a day when drinking

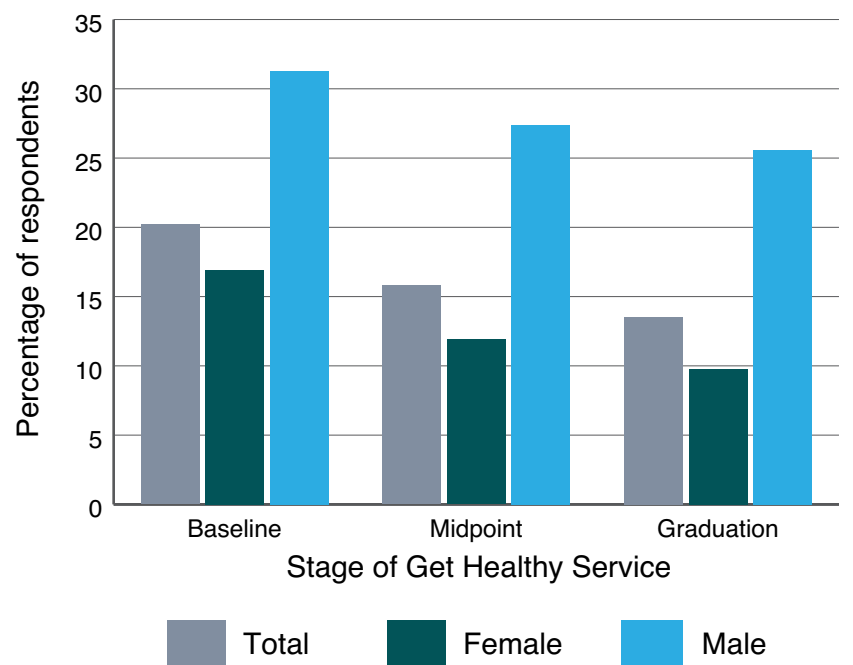

Figure 2. Get Healthy participants' immediate risk of harm, based on consumption of $>4$ standard drinks on a day when drinking

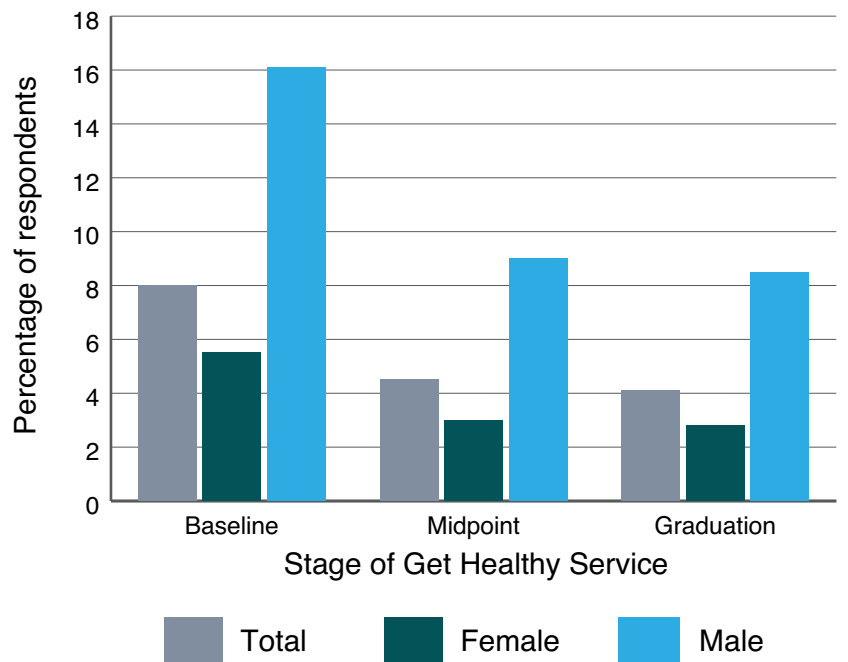

for the Alcohol Program, and there will be a further 1000 referrals from drug and alcohol professionals.

Brief interventions (Bls) are an effective tool in modifying and reducing risk behaviours, such as stopping smoking, improving nutrition and increasing physical activity. Bls are effective in primary care settings, but less so in other contexts. ${ }^{10}$ The Australian guidelines for the treatment of alcohol problems state that Bls are effective in reducing alcohol consumption for nondependent consumers of alcohol, but not for heavy or dependent drinkers, who require specialist services. ${ }^{11}$ BIs typically consist of the following 'FLAGS' steps: assessment, feedback of results, listening, advice, goals, strategies to change behaviours, referral and ongoing support. The evidence for telephone-based Bls to reduce harmful 
alcohol consumption is not currently strong, but there is evidence to suggest that Bls using telephone support and multiple sessions, such as the GHS, can be effective in reducing alcohol consumption for nondependent drinkers. ${ }^{12}$ The GHS is predominantly used by population groups who are not dependent drinkers and are motivated to engage in lifestyle-related behaviour change, making this cohort suitable for the GHS Alcohol Program.

\section{GHS Alcohol Program development}

The Alcohol Program consists of 10 coaching calls, with the goal of ceasing or reducing harmful alcohol consumption, coupled with alcohol education, management and goal-setting strategies. The Alcohol Program followed similar developmental processes for the other GHS discrete programs - type 2 diabetes prevention, healthy gestational weight gain and the Aboriginal program.

An Expert Advisory Group (EAG) was formed to guide the Alcohol Program's development. Representation included senior drug and alcohol clinicians from Sydney and South Eastern Sydney Local Health Districts, professors of public health from the universities of Newcastle and Sydney, the NSW Drug and Alcohol Network manager, a consumer, the GHS Program Manager, Director of the NSW Office of Preventive Health $(\mathrm{OPH})$, health promotion experts, and representatives from the Alcohol and Drug Information Service (ADIS), NSW Ministry of Health, and the Healthdirect and Healthways Australia services.

The EAG outlined the key components to be considered when designing the Alcohol Program, which included:

- Developing the evidence base

- Mapping referral pathways to and from the service

- Alcohol-specific coaching content

- Coach training specifications

- Considering high-risk groups, such as 16-17 year olds, pregnant women and risky drinkers

- Considering Aboriginal and other cultural groups

- Developing resources

- Promoting the program to stakeholders

- Monitoring and evaluation.

The OPH contracted a consultant to develop an implementation plan in collaboration with the EAG, contract managers for the GHS and the ADIS. The plan was sufficiently detailed to guide the implementation of the Alcohol Program.

\section{Screening GHS participants' alcohol use}

It is important that participants are appropriate for the Alcohol Program. As the GHS is not suitable for dependent drinkers, safe clinical practice requires this group to be suitably screened and referred to appropriate treatment and support services. Specialist drug and alcohol clinical advice recommended using the Alcohol Use Disorders Identification Test (AUDIT), an internationally validated tool that helps health practitioners to identify people who would benefit from reducing or ceasing risky alcohol consumption. ${ }^{13}$ The AUDIT comprises 10 questions covering recent alcohol use, alcohol-dependence symptoms and alcohol-related problems. Scores identify 'nondrinkers', 'low-risk drinkers', 'at-risk drinkers', 'high-risk drinkers' and 'likely dependent drinkers'. The AUDIT can take up to 10 minutes to complete. The shorter AUDIT Consumption (AUDIT-C) assesses frequency of drinking, typical quantity and frequency of heavy drinking. Clinical advisers recommended using AUDIT-C as the primary assessment tool for all GHS participants, to be administered at baseline by the GHS coaches. The AUDIT-C has been validated for screening in the general population ${ }^{11}$ and in Aboriginal and Torres Strait Islander populations. ${ }^{14}$

Participants scoring 1-3 in the AUDIT-C (3 items) will be offered the standard GHS, and be provided with feedback on their alcohol use. The full AUDIT (10 items) is administered when participants score 4 or higher for female and 5 or higher for male participants. Participants who score between 6 and 19 in the full AUDIT and are seeking to reduce their alcohol consumption will be enrolled in the Alcohol Program; those scoring more than 20 will be referred to the ADIS (Figure 3).

The EAG discussed the screening and referral pathways for groups such as drinkers aged 16-17 years and pregnant women. Drinkers 18 years and younger will be excluded from screening until a 12-month review of the Alcohol Program has occurred. Pregnant women scoring 1-2 in the AUDIT-C will receive abstinence advice, and pregnant women who score 3 or more on the AUDIT-C will be enrolled in the Alcohol Program and offered a referral to ADIS for additional support.

All women will receive the advice that not drinking alcohol during pregnancy and breastfeeding is the safest option.

\section{Coach training}

Providing the GHS coaches with grounding in the social and cultural context of alcohol use, and its impacts and harms is an essential aspect of implementing the Alcohol Program and providing a safe service. The $\mathrm{OPH}$ contracted a consultant with experience in delivering drug and alcohol training to deliver a customised training package. The package included training on using the AUDIT screening tool, delivering screening feedback, delivering alcohol-specific coaching and appropriately referring participants for additional support to the ADIS. The Alcohol Program's coaching calls align with current GHS coaching scripts and processes based on the FLAGS model and motivational interviewing and cognitive behaviour therapy approaches, where coaching scripts have been modified to include alcohol-specific content. Motivational interviewing is a client-centred method for improving intrinsic motivation for behaviour change by 
Figure 3. AUDIT-C screening and referral pathways for the Get Healthy Service Alcohol Program

Contact through advertisement of GHS

Participant calls GHS

\section{Contact through referral and/or advertisement of GHS Alcohol Program}

Participant calls GHS and/or is referred to the Alcohol Program

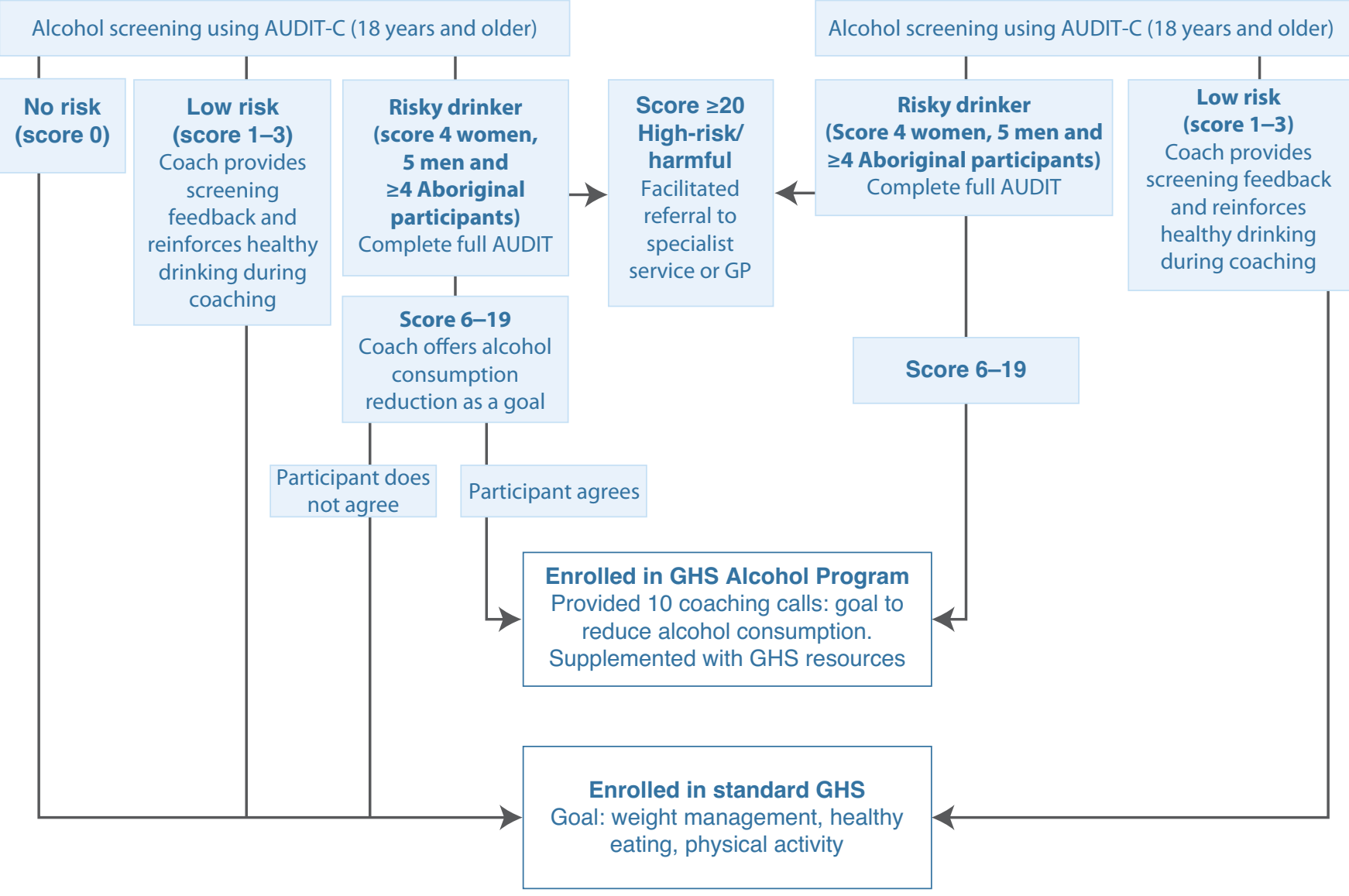

AUDIT-C = Alcohol Use Disorders Identification Test Consumption; GHS = Get Healthy Service; GP = general practitioner

exploring and resolving ambivalence. ${ }^{15}$ When applied to Bls, motivational interviewing can effectively reduce alcohol consumption, particularly in low-risk to risky drinkers. GHS coaches use motivational interviewing techniques such as open-ended questions, affirmations and reflective listening to support participants to reduce their alcohol consumption. The Alcohol Program will be supported by an alcohol-specific information booklet and a participant journey book.

\section{Promotion}

Initially, promotion of the Alcohol Program will be through existing structures and networks, such as the NSW Ministry of Health website (www.health.nsw.gov.au), the Alcohol and Drug Information Service website (www. yourroom.com.au), the GHS website (www.gethealthynsw. com.au) and other related websites. Ongoing promotion of the program will include engaging with Local Health District drug and alcohol directors, mental health directors, and population health and public health directors. Engagement with and promotion through Primary Health Networks, the nongovernment drug and alcohol sector, and Aboriginal community controlled health sector will also be pursued. To reliably assess changes in alcohol consumption, it will be important to ensure that sufficient numbers of eligible drinkers complete the 6-month coaching program.

\section{Evaluation}

The GHS routinely evaluates participants' outcomes by comparing data on health indicators (fruit and vegetable consumption, physical activity, weight and waist circumference) at baseline with the same measurements at the end of the 6-month program. These indicators, including the addition of alcohol consumption as measured by the AUDIT-C, will be measured at the start, midpoint and completion of the program. Independent validation of all GHS outcomes is conducted periodically, and will be conducted for self-reported alcohol consumption once the program referral systems and completion rates are well established. The evaluation of the Alcohol Program will be an opportunity to add to the evidence on telephone-based Bls to reduce risky alcohol consumption. 


\section{Discussion}

Drug and alcohol clinical advice has been an essential component in determining appropriate groups for inclusion in the development of the Alcohol Program. The GHS is not a suitable service for people with alcohol dependence problems and may be dangerous for dependent drinkers to reduce or cease alcohol consumption, which may precipitate withdrawal. ${ }^{11}$ To ensure clinical safety, supported referral processes have been developed. The facilitated referral process ensures participants are linked with a suitable drug and alcohol service, such as the ADIS. This pathway will be used to refer pregnant women, underage drinkers and high-risk drinkers scoring 20 or more on the AUDIT-C tool.

This is the first time a telephone-based alcohol reduction program has been offered in NSW, and the initial focus of evaluation will be on the acceptability of the program to participants and how well the program operates. ${ }^{16}$ The Alcohol Program's viability should be demonstrated before focusing on program outcomes. However, based on the best available evidence, our approach has a strong likelihood of success with nondependent alcohol drinkers. ${ }^{12,15}$

\section{Conclusions}

The Alcohol Program was developed following a considered approach using the best available evidence, an EAG and adapting evidence based materials. The Alcohol Program has the potential to provide effective coaching to adults to voluntarily reduce short-term and long-term risky alcohol consumption. The Alcohol Program is a welcome addition to the range of alcohol $\mathrm{BI}$ programs in Australia.

Future work will include the development of an Aboriginal-specific participant journey book to support Aboriginal participants entering the Alcohol Program. Future research will examine the impact of telephonebased coaching on reducing risky alcohol consumption.

\section{Competing interests}

None declared

\section{Author contributions}

All authors contributed to the design and writing of the manuscript, and were involved in the development of the program materials.

\section{References}

1. National Health and Medical Research Council. Australian guidelines to reduce health risks from drinking alcohol. Canberra: Commonwealth of Australia; 2009 [cited 2016 Aug 12]. Available from: www.nhmrc.gov.au/_files_nhmrc/publications/ attachments/ds10-alcohol.pdf

2. Laslett AM, Mugavin J, Jiang H, Manton E, Callinan S, MacLean S, Room R. The hidden harm: alcohol's impact on children and families. Canberra: Foundation for Alcohol Research and Education; 2015 [cited 2016 Aug 12]. Available from: fare.org.au/wp-content/ uploads/research/01-ALCOHOLS-IMPACT-ONCHILDREN-AND-FAMILIES-web.pdf

3. Collins DJ, Lapsley HM. The costs of tobacco, alcohol and illicit drug abuse to Australian society in 2004-05. Canberra: Commonwealth of Australia; 2008 [cited 2016 August 12]. Available from: www.health.gov.au/ internet/drugstrategy/publishing.nsf/Content/34F55AF632 F67B70CA2573F60005D42B/\$File/mono64.pdf

4. Audit Office of New South Wales. Cost of alcohol abuse to the NSW Government. Sydney: Audit Office of New South Wales; 2013 [cited 2016 Aug 14]. Available from: www.audit.nsw.gov.au/ArticleDocuments/281/01_Cost_ of_alcohol_abuse_NSW_Government_Full_Report.pdf. aspx?Embed $=Y$

5. New South Wales Ministry of Health. NSW state health plan: towards 2021. North Sydney, NSW Ministry of Health; 2014 [cited 2016 Aug 14]. Available from: www.health.nsw.gov.au/statehealthplan/Publications/ NSW-State-Health-Plan-Towards-2021.pdf

6. Australian Institute of Health and Welfare. National drug strategy household survey 2013. Canberra: AlHW; 2014 [cited 2016 Aug 12]. Available from: www.aihw.gov.au/ WorkArea/DownloadAsset.aspx?id=60129549848

7. HealthStats NSW. Alcohol drinking in adults. Centre for Epidemiology and Evidence, NSW Ministry of Health; 2016 [cited 2016 Mar 1]. Available from: www.healthstats.nsw.gov.au.

8. O'Hara BJ, Phongsavan P, McGill B, Maxwell M, Ahmed N, Raheb S, Bauman AE. NSW Get Healthy Information and Coaching Service: the first five years 2009-2013. Sydney: NSW Ministry of Health; 2014 [cited 2016 Aug 12]. Available from: www.gethealthynsw.com. au/assets/pdf/medicalprofessionals/resources/Get_ Healthy_Service_Evaluation_Report_WEB_version.pdf

9. Cordery D. Get Healthy Information \& Coaching Service: alcohol module. North Sydney: NSW Ministry of Health; 2015.

10. Moyer A, Finney JW, Swearingen CE, Vergun P. Brief interventions for alcohol problems: a meta-analytic review of controlled investigations in treatmentseeking and non-treatment seeking populations. Addiction. 2002;97(3):279- 92. 
11. Haber P, Lintzeris N, Proude E, Lopatko O. Guidelines for the treatment of alcohol problems. Canberra: Commonwealth of Australia; 2009 [cited 2016 Aug 14]. Available from: www.health.gov.au/internet/main/ publishing.nsf/ontent/OFD6C7C289CD31C9CA257BF000 1F96BD/\$File/AustAlctreatguidelines\%202009.pdf

12. Bischoff G, Grouthes JM, Reinhardt S, Meyer C, John U, Rumpf HJ. Evaluation of a telephone-based stepped care intervention for alcohol-related disorders: a randomised controlled trial. Drug Alcohol Depend. 2008;93(3):244-51.

13. Babor TF, Higgins-Biddle JC, Saunders JB, Monteiro MG. AUDIT: the alcohol use disorders identification test. Guidelines for use in primary care. Geneva: World Health Organisation; 2001 [cited 2016 Aug 14]. Available from: apps.who.int/iris/bitstream/10665/67205/1/WHO_MSD_ MSB_01.6a.pdf
14. Calabria B, Clifford A, Shakeshaft AP, Conigrave KM, Simpson L, Bliss D, Allan J. Identifying Aboriginalspecific AUDIT-C and AUDIT-3 cutoff scores for at-risk, high-risk, and likely dependent drinkers using measures of agreement with the 10-item Alcohol use Disorders Identification Test. Addict Sci Clin Pract. 2014;9(1):17.

15. Vasilaki El, Hosier SG, Cox WM. The efficacy of motivational interviewing as a brief intervention for excessive drinking: a meta-analytic review. Alcohol Alcohol. 2006;41(3):328-35.

16. Nutbeam D, Bauman A. Evaluation in a nutshell: a practical guide to the evaluation of health promotion programs. Sydney: McGraw-Hill Australia; 2006.

\section{Copyright: (c) (i) (2)}

(c) 2016 Moore et al. This article is licensed under the Creative Commons Attribution-NonCommercial-ShareAlike 4.0 International Licence, which allows others to redistribute, adapt and share this work non-commercially provided they attribute the work and any adapted version of it is distributed under the same Creative Commons licence terms. See: www.creativecommons.org/licenses/by-nc-sa/4.0/ 